BULl. AUSTRAL. MATH. SOC.

VOL. 33 (1986), 21-36.

\title{
INVARIANT QUADRATIC FORMS ON FINITE DIMENSIONAL
}

\section{LIE ALGEBRAS}

\author{
Karl H. Hofmann and Verena S. Keith
}

\begin{abstract}
Trace forms have been well studied as invariant quadratic forms on finite dimensional Lie algebras; the best known of these forms is the Cartan-Killing form. All those forms, however, have the ideal $[L, L] \cap R$ (with the radical $R$ ) in the orthogonal $L^{\perp}$ and thus are frequently degenerate. In this note we discuss a general construction of Lie algebras equipped with non-degenerate quadratic forms which cannot be obtained by trace forms, and we propose a general structure theorem for Lie algebras supporting a non-degenerate invariant quadratic form. These results complement and extend recent developments of the theory of invariant quadratic forms on Lie algebras by Hilgert and Hofmann [2], Keith [4], and Medina and Revoy [7].
\end{abstract}

\section{Introduction}

A bilinear form $q: L \times L \longrightarrow F$ on a Lie algebra $L$ over a field $F$ of characteristic 0 is said to be invariant if and only if $q([x, y], z)=q(x,[y, z])$ for all $x, y, z \in L$ and to be a quadratic form if and only if it is symmetric, that is satisfies $q(x, y)=q(y, x)$. If $\pi: L \longrightarrow g l(V)$ is a representation of a Lie algebra $L$ on a finite

Received 11 April 1985.

Copyright Clearance Centre, Inc. Serial-fee code: 0004-9727/86 $\$ A 2.00+0.00$. 
dimensional $F$-vector space $V$, then $q(x, y)=\operatorname{tr} \pi(x) \pi(y)$ is a wellknown invariant quadratic form, called the trace form (associated with $\pi$ ). If, in particular, $V$ is the underlying vector space of $L$ and $\pi$ is the adjoint representation, then $q$ is called the Cartan-Killing form of $L$. The orthogonal $L^{\perp}=\{x \in L: q(L \times\{x\})=\{0\}\}$ with respect to any trace form will contain $[L, L] \cap R$, where $R$ denotes the radical of $L$ (for this and other background information see for example Bourbaki [1]); in fact the characteristic ideal $[L, L] \cap R$ is the intersection of the orthogonals of all trace forms. Whenever $[L, L] \cap R \neq 0$, therefore, each trace form must be degenerate. The trivial example of an abelian Lie algebra, however, shows that there may very well be non-degenerate invariant quadratic forms which are not trace forms.

Non-degenerate invariant quadratic forms, curiously, have only recently been made the object of systematic studies. The general theory was, in fact, developed independently by Medina and Revoy [7] and in the dissertation of V.S. Keith [4]. The purpose of this note is to contribute to the theory of Lie algebras with invariant quadratic forms by the formulation of a certain construction method and a general structure theorem, which complements a theorem of Medina and Revoy [7].

At first sight, it is not at all clear whether Lie algebras with invariant quadratic forms exist in abundance outside the classically studied domains of semisimple Lie algebras (where the Cartan-Killing form will dol and the compact real Lie algebras (which are characterized by the fact that they carry invariant positive definite quadratic forms). It was, however, shown in Keith [4] and Medina and Revoy [7] that through appropriate extension processes, Lie algebras with non-degenerate invariant quadratic forms could be built from simpler building blocks and that, in this fashion, solvable algebras could be obtained. It was noted by Guts and Levichev [3], Medina and Revoy [8], Keith [4,pp.69,70] that the so-called oscillator algebra, a four-dimensional real Lie algebra arising in the quantum mechanical description of a harmonic oscillator, carries an invariant non-degenerate quadratic form of Lorentz type, which then led to the discovery of a whole countable series of solvable lie algebras which support invariant Lorentzian forms (and are irreducible in a suitable sensel; see also Hilgert and Hofmann [2] in this context. 
Medina and Revoy show in [7] that all Lie algebras can be obtained by an algorithm proceeding through a repeated extension process which they call "double extension" $[7, \mathrm{p} .73]$ and which Keith calls bi-extension $[4, \mathrm{p} .65]$. It is, however not easy to see what particular Lie algebras the algorithm will lead to after several steps. Therefore, it is relevant to have available explicit construction processes which will lead to certain identifiable types of algebras exhibiting the complexity that lie algebras with invariant bilinear forms can have despite the restraining influence exerted by the presence of a non-degenerate invariant quadratic form on the ideal structure of the algebra, as was shown by Keith [4] and Medina and Revoy [7]. We therefore propose a basically simple construction which, nevertheless, will produce for example nilpotent Lie algebras of arbitrarily high class of nilpotency with non-degenerate bilinear forms and mixed Lie algebras (that is Lie algebras which are neither solvable nor semisimplel of considerable degree of complexity.

Indeed let $(L, q)$ be a Lie algebra with an invariant quadratic form. We consider now a finite dimensional commutative and associative $F$-algebra $(A, p)$ together with a quadratic form $p$ which is invariant in the sense that $p(x y, z)=p(x, y z)$ for all $x, y, z \in A$. On the $F$-tensor-product $A \otimes_{F} L$, which is none other than a ground ring extension of $L$, we have a Lie algebra structure given by the bracket $[a \otimes x, b \otimes y]=a b \otimes[x y]$ and an invariant quadratic form $p \otimes q$ given by $(p \otimes q)(a \otimes x, b \otimes y)=p(a, b) q(x, y)$. We then have the following

PROPOSITION A. For a finite dimensional associative, commutative algebra (A,p) with invariant quadratic form and a finite dimensional lie algebra $(L, q)$ with invariant quadratic form, the tensor product over the ground field $(A \otimes L, p \otimes q)$ is a Lie algebra with an invariant quadratic form. If both $p$ and $q$ are non-degenerate, then $p \otimes q$ is non-degenerate. If $A$ is nilpotent, then $A \otimes L$ is nilpotent (regardiess of $L)$. If $A$ has an identity and $L$ is not solvable, then $A \otimes L$ is a mixed Lie algebra, provided $A$ is not semisimple.

We shall give examples of commutative associative nilpotent algebras of arbitrary nilpotent length with non-degenerate invariant quadratic forms with the aid of semigroup algebras. Thus we will get, upon taking $(L, q)$ simple with the Cartan-killing form, the following 
COROLLARY B. Given any natural number $n$, there exist nilpotent Lie algebras of nilpotency class $n$ with a non-degenerate invariant quadratic form.

EXAMPLE. Let $S$ be a simple algebra with its Cartan-Killing form $Q$. On $S^{n}$ we define $\left[\left(x_{1}, \ldots, x_{n}\right),\left(y_{1}, \ldots, y_{n}\right)\right]=\left(z_{1}, \ldots, z_{n}\right)$ with $z_{k}=\sum_{j=1}^{k-1}\left[x_{j}, y_{k-j}\right]$, and $q\left(\left(x_{1}, \ldots, x_{n}\right),\left(y_{1}, \ldots, y_{n}\right)\right)=\sum_{j=1}^{n} Q\left(x_{j}, y_{n-j}\right)$.

If we take $A$ to have an identity and $L$ to be a semisimple lie algebra, we shall be able to construct mixed Lie algebras with invariant non-degenerate forms:

COROLLARY C. Given a natural number $n$ and a semisimple lie algebra $S$, there is a lie algebra $L$ with a nilpotent radical $R$ of length $n$ such that $L / R=S$ and $[L, R]=R$ such that $L$ supports $a$ non-degenerate invariant quadratic form which is degenerate on no proper ideal.

Upon taking $A$ to be the algebra of dual numbers, that is the two dimensional algebra spanned by 1 and $e$ with $e^{2}=0$ we can introduce on $A$ an invariant quadratic form $p_{r S}$ for each pair of scalars $r, s \in F$ by setting $p_{r s}\left(u+v e, u^{\prime}+v^{\prime} e l=r\left(u v^{\prime}+v u^{\prime}\right)+s u u^{\prime}\right.$; this form is non-degenerate if and only if $r \neq 0$. Then for any Lie algebra $(L, q)$ with an invariant quadratic form, $\left(A \otimes L, p_{r s} \otimes q\right)$ is a Lie algebra with invariant quadratic form by Proposition $A$, and its form is non-degenerate if and only if $r \neq 0$ and $q$ is non-degenerate. The algebra $A \otimes L$ is isomorphic to the semidirect product $|L| \times L$ with $|L|$ denoting the adjoint $L$-module defined on the underlying vector space of $L$; the Lie bracket is simply $[(a, x[,(b, y)]=c[a, y]+[x, b],[x, y])$. The invariant form $p_{r s} \otimes q$ corresponds to the form $q_{r s}$ on $|L| \times L$ given by $q_{r s}((a, x),(b, y))=r(q(a, y)+q(x, b))+s q(x, y)$.

DEFINITION D. For a Lie algebra $L$ we will call $1 L 1 \rtimes L$ the inflation $I C L L$ of $L$. If $Q$ is an invariant bilinear form on $L$ and $P$ is an invariant quadratic form on $L$ then we define an invariant quadratic form $q$ on $I(L)$ by $q((a, x),(b, y))=Q(a, y)+Q(b, x)+P(x, y)$ 
and we call $(I(L), q)$ the inflation of $L$ with respect to the forms $P$ and $Q$. We will also say $q$ is of type $q_{P Q}$.

This example was given by Keith $[4$, p.30] and by Medina and Revoy [7, p.87] with the co-adjoint module on the dual $L^{*}$ instead of $|L|$. (If $q$ is non-degenerate on $L$, then $|L|$ and $L^{*}$ are isomorphic $L$-modules anyhow:) The tangent bundle of any Lie group is a Lie group whose Lie algebra is the inflation of the Lie algebra of the group. What is relevant for us regarding inflations is that they occur prominently in the structure of mixed algebras with invariant quadratic forms.

For simple algebras, Medina and Revoy established the following result by an appropriate interpretation of Schur's Lemma:

PROPOSITION E. (Medina and Revoy [6,pp.72 and 73].)

Suppose that $(S, q)$ is a simple Lie algebra over a field of characteristic 0 and $q$ any invariont quadratic form, then there is a finite field extension (G:F) such that $S$ is obtained from a G-algebra $S_{G}$ by restriction of scalars, and from the Cartan-Killing form $k_{G}$ of $S_{G}$ one obtains $q=f \circ k_{G}$ with an $F$-linear form $f: G \longrightarrow F$.

DEFINITION F. We call a quadratic form $q$ on a simple lie algebra obtained in this fashion a modified killing form.

A modified Killing form is either zero or non-degenerate. The proof of Medina and Revoy actually shows that a similar statement holds for any invariant bilinear form (with an appropriate field extension). This enables us to derive

COROLLARY G. An invariant quadratic form on the inflation $I(S)$ of a simple Lie algebra is necessarily of type $q_{P Q}$ with modified Killing forms $P$ and $Q$ of $S$.

From Proposition E one deduces readily that every semisimple lie algebra is an orthogonal dixect sum of simple ones on each of which the form induces a scalar multiple of a modified Killing form. In a similar vein, Corollary $G$ implies that an inflation of a semisimple Lie algebra is an orthogonal direct sum of inflations of simple Lie algebras so that on each sumnand the form induces a form of type $q_{P Q}$ with modified killing forms $P, Q$. 
With these pieces of information we can now appreciate the structure theorem for mixed Lie algebras with a non-degenerate quadratic form. Since every such algebra splits into an orthogonal direct sum of summands neither of which contains an ideal on which the form is non-degenerate (Medina and Revoy $[7, \mathrm{p}, 71]$ ) it suffices to consider algebras $(L, q)$ in which $q](I \times I)$ is degenerate for each proper ideal $I$; we shall call such pairs $(L, q)$ irreducible.

THEOREM $H$. Let $(L, q)$ be an irreducible pair of a finite dimensional Lie algebra $L$ over a field of characteristic 0 and a nondegenerate invariant quadratic form. Let $R$ be the radical of $L$ and $S$ cony Levi complement for $R$ in $L$. Let $R^{\perp}=\{x \in L: q(R \times\{x\})=\{0\}\}$ denote the orthogonal of $R$. Then the following conclusions hold, provided $L$ is not semisimple:

(1) $R^{\perp}$ is contained in the centre of $R$ and as an L-module under the adjoint action is isomorphic to the quotient module $L / R$.

(2) The subalgebra $A=S+R^{\perp}$ is isomorphic to the inflation of $S$, and the restriction $q \mid(A \times A)$ is non-degenerate on $A$, so that $A$ is an orthogonal direct sum of inflations $\left(I\left(S_{j}\right), q_{j}\right)$ of the simple factors $s_{j}$ with form $q_{j}=q_{P_{j} Q_{j}}$ with modified Kizling forms $P_{j}$ and $Q_{j}$ on $S_{j}$.

The ideal theory of Lie algebras with non-degenerate invariant quadratic forms tells us that $q$ will induce on $R / R^{\perp}$ a non-degenerate quadratic form, so that the theory applies to $R / R^{\perp}$ and shows that it is a central bi-extension of the nilpotent algebra $[R, R] /[R, R]^{\perp}$ (see Section 1 below for the concept of a bi-extension). The situation is illustrated in Figure 1 .

Theorem $\mathrm{H}$ overlaps and complements Theorem 6.17, p.88 of Keith [4] and Theorem 5.1 on p.79 of Medina and Revoy [7].

The semisimple case is clear: A semisimple Lie algebra with nondegenerate quadratic form is an orthogonal direct sum of simple algebras with modified killing forms.

The examples resulting from Corollary $C$ show that a substantial improvement of Theorem $\mathrm{H}$ is not to be expected. 


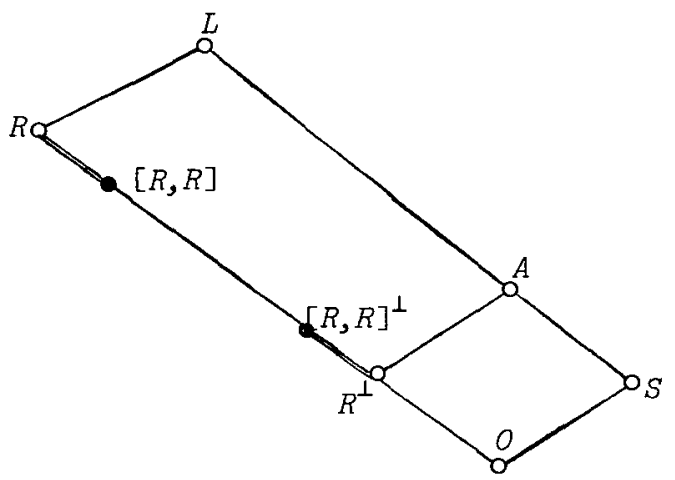

Figure 1.

The remainder of the paper is organized as follows: section 1 provides the necessary background material from the general theory for which we refer to Keith [4] and Medina and Revoy [7]. Section 2 presents the proofs of the results described in this introduction.

1. The general theory of Lie algebras with invariant quadratic forms Let $L$ be a finite dimensional Lie algebra over a field $F$ of characteristic 0 . If $M$ is an $L$-module (see [1]), then a bilinear form $q: M \times M \longrightarrow F$ is called invariant if and only if $q(x, m, n)+q(m, x . n)=0$ for all $x \in L$ and $m, n \in M$. Whatever we say in such a situation applies in particular to the case of the adjoint module $M=|L|$ of $L$ if $q$ is an invariant bilinear form on $L$. The dual module $M^{*}$ is defined on the vector space dual of $M$ by $\langle x . \omega, m\rangle=-\langle\omega, x . m\rangle$. We note $[4, p .18]$

1.1. REMARK. An $L$-module $M$ is isomorphic to its dual module if and only if it supports an invariant non-degenerate bilinear form.

1. 2. DEFINITION. If $M$ is an $L$-module with an invariant bilinear form $q$, and $X \in M$, we set $X^{\perp}=\{m \in M: q(x \times\{m\})=\{0\}\}$. If $N$ is a submodule of $M$ we write $L . N$ for the submodule of linear combinations of elements $x . m$ with $x \in L$ and $m \in M$, and $N_{L}$ for the submodule $\{m \in M: L . m \subseteq N\}$. 
From the invariance of $q$ it is clear that $N^{\perp}$ is a submodule, too. There are no particular difficulties in proving the following proposition [4,p. 18 ff.]:

1.3. PROPOSITION. FOr any L-module $M$ with an invariant non-degenerate bilinear form the function $N \rightarrow N^{\perp}$ is a containment reversing lattice anti-automorphism of the lattice of submodules of $M$ which is involutive (that is satisfies $N^{\perp \perp}=N$ ). It maps $L . N$ to $N_{L}$ and, more generally, the descending series of modules $N^{k}$ (defined inductively by $N^{k}=L . N^{k-1}, k=1,2, \ldots$ with $\left.N^{0}=N\right)$ to the ascending series of modules $N_{k}$ (defined inductively by $N_{k} / N_{k-1}=\left(N / N_{k-1}\right)_{L}, k=1,2, \ldots$ with $\left.N_{0}=\{0\}\right)$. For each submodule $N$, the dual module $N^{*}$ is naturally isomorphic to $M / N^{\perp}$.

If we let $M=|L|$, we obtain the following corollary (see $[4, \mathrm{p} .32]$ and $[6], p .69)$ :

1.4. COROLLARY. In any Lie algebra with an invariant non-degenerate quadratic form, the function $J \rightarrow J^{\perp}$ is an involutive lattice antiautomorphism of the lattice of ideals of $L$, mapping the commutator algebra $[L, L]$ to the centre $Z(L)$ and, more generally, the descending central series to the ascending central series.

By induction, one readily calculates the following.

1.5. COMPLEMENT. Under the circunstances of 2.4 , the function $J \leftrightarrow J^{\perp}$ maps the commutator series $L^{[k]}=\left[L^{[k-1]}, L^{[k-1]}\right], L^{[0]}=L$ to the ascending series of ideals defined recursively by $k^{0}(L)=0$, $K^{k}(L)=\left\{x \in L:\left[L^{[k-1]}, x\right] \subseteq K^{k-1}(L)\right\}$.

Through these results, the presence of an invariant non-degenerate bilinear form on a Lie algebra forces the lattice of ideals to exhibit strong symetry. This observation frequently allows us to determine that a given Lie algebra is incapable of supporting a non-degenerate invariant bilinear form. Such is the case for example for the three-dimensional nilpotent Heisenberg algebra. Lie algebras of small dimension are usually inspected quickly for the required symmetry of their ideal lattice (see $[4],[6])$. 
The following simple observation is still often useful. [4,p.79]: 1.6. LEMMA. a) If $H$ and $K$ are subalgebras of a Lie algebra with an invariant bilinear form and if $[H, K]=\{0\}$, then $[H, H] \subseteq K^{\perp}$ and $[K, K] \subseteq H^{\perp}$.

b) If, in addition, $H$ is perfect, that is satisfies $[H, H]=H$, then $H \subseteq K^{\perp}$.

1.7. LEMMA. b) Let $J$ be an ideal in a Lie algebra with a nondegenerate invariant quadratic form. Then $\left[J, J^{\perp}\right]=0$, and $J$ is commitative if and only if $J \subseteq[L, J]^{\perp}$. In particutar, $J$ is conmutative if $J \subseteq J^{\perp}$.

If the restriction of a non-degenerate invariant quadratic form to an ideal $J$ is non-degenerate, then the algebra is the orthogonal direct sum of the ideals $J$ and $J^{\perp}$. The general case that this restriction is degenerate is described in the following summaxy whose assertions are readily established:

1.8. PROPOSITION. Let $J$ be an ideal in a lie algebra with a nondegenerate invariant bilinear form $q$. On the factor algebra $\bar{L}=\left(J+J^{\perp}\right) /\left(J \cap J^{\perp}\right)$ the prescription $\bar{q}(x+I, y+I)=q(x, y)$ with $I=J \cap J^{\perp}$ defines unambiguously a non-degenerate invariant quadratic form. The ideal $I$ is central in $J+J^{\perp}$ and as an L-module is isomorphic to $\left(L /\left(J+J^{\perp}\right)\right) *$ (see Figure 2).

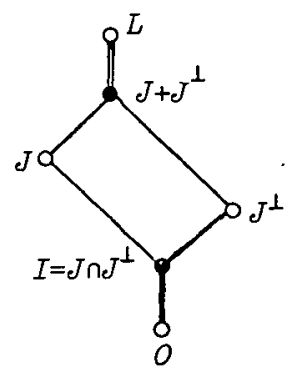

Figure 2. 
This calls for a definition which one finds in $[4, p .65]$ and in $[6, \mathrm{p} .73$ ff. $]$.

1.9. DEFINITION. A Lie algebra $(L, q)$ with an invariant quadratic form is called a bi-extension of a Lie algebra $(M, p)$ with invariant quadratic form $p$ if and only if $L$ contains an ideal $J$ with $J^{\perp} \subseteq J$ such that there is an isomorphism $f: M \longrightarrow J / J^{\perp}$ yielding $p(m, n)=q(f(m), f(n))$.

If $q$ is non-degenerate, then $p$ must be non-degenerate and $J^{\perp}$ is central in $J$ by 2.8. We say that $(L, q)$ is a central bi-extension of $(M, p)$ if $J^{\mathcal{L}}$ is central even in $L$.

Explicit constructions of bi-extensions in terms of functions and functional equations were given by Keith in $[4, p .56 \mathrm{ff}]$ and by Medina and Revoy $[7, p .73 \mathrm{ff}$.$] . The latter proved the following result which shows$ that Lie algebras with invariant non-degenerate forms can be successively constructed via bi-extensions:

1.10. THEOREM (Medina and Revoy).

The class of Lie algebras supporting a non-degenerate quadratic form is the smallest class containing all abelian and all simple algebras and which is closed under the formation of direct products and bi-extensions $[7, p .80$, Thm. 5.2].

Section 2. Proofs and supplements.

If $A$ is a commutative and associative algebra over the field $F$ and $L$ a Lie algebra over $F$, then $A \otimes_{F} L$ with the multiplication given in the introduction is simply the ground ring extension of $L$ (see [1]). The assertions of Proposition A on the tensor product $p \otimes q$ of two invariant forms on $A$ and $L$, respectively are straightforward. We define recursively $A^{[0]}=A$ and $A^{[k]}=A A^{[k-1]}$, also $L^{[0]}=L$ and $L^{[k]}=\left[L, L^{[k-1]}\right], n=1,2, \ldots$ Then the following is straightforward: 2.1. LEMMA. $(A \otimes L)^{[k]}=A^{[k]} \otimes L^{[k]}, k=0,1,2, \ldots$

2.2. COROLLARY. If at least one of $A$ and $L$ is nilpotent, then $A \otimes L$ is nizpotent. 
2.3. COROLLARY. If $L=[L, L]$, then $(A \otimes L)^{[k]}=A^{[k]} \otimes L$ and if $A^{[n+1]}=\{0\}$ for the first time, then $A \otimes L$ is nizpotent of class $n$.

If $A$ has an identity and $L$ a Levi complement $S$ for its radical, then $1 \otimes S$ is a semisimple subalgebra of $A \otimes L$. If $A$ has a radical $N$, then $N$ and thus $N \otimes L$ is nilpotent, whence $A \otimes L$ is a mixed Lie algebra. This completes the proof of Proposition $A$ of the introduction.

Now we have to construct a nilpotent commutative and associative algebra of given nilpotent length $n$. Let $S$ be a multiplicatively written semigroup with zero $z$. The semigroup algebra $F[S]$ is the vector space freely generated by the elements of $S$ and subject to the multiplication obtained by extending that of $S$ linearly. We note that $F \cdot z$ is an ideal and set $F_{O}[S]=F[s] / F . z$. We may consider $F_{O}[S]$ as the vector space freely generated by $S \backslash\{z\}$ with a multiplication inducing that of $S$ when $z$ and 0 are identified. We will call $F_{0}[S]$ the reduced semigroup algebra. If $S$ is a nilpotent semigroup, that is $S^{n}=\{0\}$ for some $n$, then $F_{0}[S]$ is a nilpotent algebra, which is also commutative if $S$ is commutative. If $S$ is finite, then $F_{0}[S]$ is finite-dimensional; we note that the matrix of semigroup elements (st) $s, t \in S \backslash\{z\}$ is also called the (reduced) multiplication table of the semigroup.

2.4. LEMMA. Let $S$ be a finite semigroup with zero $z$ and $f: F_{0}[z] \longrightarrow F$ any function. Extend $f$ linearly to a function $f: F_{0}[S] \longrightarrow F$. Then the bilinear form $p$ defined on $F_{0}[S]$ by $p(x, y)=f(x y)$ is invariant. If the matrix

$$
(f(s t))_{s, t \in S \backslash\{z\}}
$$

is non-singular, then $p$ is non-degenerate. The form $p$ is quadratic if $S$ is commutative.

Proof. The invariance of $p$ is trivial because of $p(x y, z)=f(x y z)=p(x, y z)$. If $S$ is commutative, then $p$ is clearly symetric. Now suppose that $p(x, y)=0$ for all $x \in F[S]$. Then, in particular $f(s y)=0$ for all $s \in S \backslash\{z\}$. If we set 
$y=\left\{\left\{r_{t} \cdot t: t \in S \backslash\{z\}\right\}\right.$ with $r_{t} \in F$, then $\left(r_{t}\right) t \in S \backslash\{z\}$ is the solution of a system of homogeneous linear equations with coefficient matrix $(f(s t))_{s, t} \in S \backslash\{z\}$. If this coefficient matrix is non-singular, then the solution must be zero and we conclude $y=0$. Thus $p$ is non-degenerate in this case.

The simplest nilpotent semigroup is the semigroup $\mathbb{I}_{k}=\{1, \ldots, k+1\}$ with the multiplication $x \circ y=\min \{s+t, k+1\}$. Then $z=k+1$ is the zero of the semigroup and $F_{0}\left[\mathbb{N}_{k}\right]$ is a $k$-dimensional nilpotent commutative algebra with nilpotent length $k$.

2.5. LEMMA. If $S=\mathbb{N}_{n}$ and $f:\{1, \ldots, n\} \longrightarrow F$ is the constant function with value 1 , then the quadratic form $p$ constructed in Lemma 2.4 is non-degenerate on $F_{0}\left[N_{n}\right]$.

Proof. We note $f(s \circ t)=1$ for $s+t \leq n+1$, else 0 . Hence the matrix $(f(s \circ t))_{s, t=1, \ldots, n}$ is clearly non-singular. The assertion then follows from Lemma 2.4 .

Obviously we could have taken other functions $f$ instead, for example the one taking the value $l$ on $n+1$, the value 0 elsewhere. In view of Proposition $A$ and Lemma 2.5, we finish the proof of Corollary $B$ readily by taking $L=F_{0}\left(\mathbb{N}_{n}\right) \otimes S$ with any simple Lie algebra, for example $s l(2, F)$ and by considering $p \otimes k$ with the Cartan-Killing form on $S$ as quadratic form. The example in Corollary B of the Introduction is just an isomorphic rendering of this example here.

If we consider the semigroup $\mathbb{N}_{0 k}=\{0,1, \ldots, k+1\}$ with $x \circ y=\min \{x+y, k+1\}$, then $F_{O}[\mathbb{N} O K=$ is a commutative algebra $A$ of dimension $k+1$ with identity and radical $F_{0}\left[\mathbb{N}_{k}\right]$. Again by the process of Lemmas 2.4 and 2.5.we find invariant non-degenerate quadratic forms $p$ on $A$, and if we let $S$ again be a simple lie algebra, then $p \otimes k_{S}$ is a non-degenerate quadratic form on $L=A \otimes S$. The radical $R$ of $L$ is $F_{0}\left[\boldsymbol{N}_{k}\right] \otimes S$, a nilpotent algebra of class $k$. The subalgebra $1 \otimes S$ is a Levi complement. The relation $[L, R]=R$ is readily verified. Thus Corollary $\mathrm{C}$ of the Introduction is proved. 
Now we consider the inflation $I(S)=|S| \rtimes S$ of a simple lie algebra $S$ and let $q$ be an invariant quadratic form on $I(S)$.

2.6. LEMMA. There are modified Killing forms $P, Q$ on $S$ (see Definition $F$ in the Introduction) such that $q$ is of type $q_{P Q}$ (see Definition $D$ in the Introduction).

Proof. The only ideals of $I(S)$ are $\{0\}, I(S)$ and $R=S \times\{0\}$. If $L^{\perp}=L$, then $q=0$ and the assertion holds with $P=Q=0$. If $L^{\perp}=R$, then by $1.8, q$ induces on $S \cong I(S) / R$ a non-degenerate quadratic form which by Medina's and Revoy's Proposition must be a modified Killing form $k$ on $S$. Hence the assertion is true with $P=k$ and $Q=0$. Finally assume that $L^{\perp}=\{0\}$, that is that $q$ is non-degenerate. Then $R \quad$ is an ideal whose codimension $\operatorname{dim} I(S) / R$ is $\operatorname{dim} R^{*}=\operatorname{dim} S$ by the last statement of 1.3. But $R$ itself is the only ideal with this dimension, and so $R^{\perp}=R$. This implies that there are bilinear maps $P, Q: S \times S \longrightarrow F$ such that $q((u, x),(v, y))=Q(u, y)+Q(v, x)+P(x, y)$ with a symmetric $P$ in view of the symmetry of $q$. Since $q([(u, 0),(0, x)],(0, y))=Q([u, x], y)$ and $q((u, 0),[(0, x),(0, y)])$ $=Q(u,[y, x])$, the invariance of $q$ shows the invariance of $Q$. By the extension of the Proposition of Medina and Revoy in the Introduction to invariant bilinear forms on simple algebras, we conclude that $Q$ must be a modified killing form on $S$. Iikewise $P$ must be a modified killing form on $S$.

2.7. LEMMA. Let $I(L)$ be an inflation of a semisimple algebra $L$. Then $I(L)$ is a direct sum of inflations $I\left(S_{j}\right)$ of the simple factors of $s_{j}$. Moreover, if $q$ is an invariant non-degenerate quadratic form on $I(S)$, then this sum is orthogonal.

Proof. Let $L=S_{1} \oplus \ldots \oplus S_{n}$ be the unique direct sum decomposition of $L$ into simple factors. Then the adjoint module $|L|$ is the direct sum of the submodules $\left|S_{j}\right|$, and it follows readily from the definition of the inflation, that $I(L)$ is the direct sum of the inflations $I\left(S_{j}\right)$ (up to a natural isomorphism). Furthermore each of the summands $I\left(S_{j}\right)$ is perfect as is every partial sum of them; hence 
Lemma 1.6 applies to show that the direct sum decomposition is orthogonal with respect to any non-degenerate invariant quadratic form.

2.8 LEMMA. Let $L$ be a Lie algebra in which each ideal $J \neq 0$ satisfies $J \cap J^{\perp} \neq\{0\}$ with respect to a given invariant non-degenerate quadratic form. Then the radical $R$ satisfies $R^{\perp} \subset R$.

Moreover, $R^{\perp}$ is central in $R$.

Proof. The factor algebra $\left(R+R^{\perp}\right) / R$ is semisimple and isomorphic to $R^{\perp} /\left(R \cap R^{\perp}\right)$, hence $R \cap R^{\perp}$ is the radical of $R^{\perp}$ and we find a Levi complement $H$ of $R \cap R^{\perp}$ in $R^{\perp}$ so that $R^{\perp}=\left(R \cap R^{\perp}\right)+H$ and thus $R+R^{\perp}=R+H$. In order to prove the Lemma we must show $H=\{0\}$. Now $[R, H]=[R,[H, H]]$ (since $H$ is perfect) $\subseteq[[R, H], H]$ (by the Jacobi identity) $\subseteq\left[\left[R, R^{\perp}\right], R^{\perp}\right] \subseteq\left[R, R^{\perp}\right]=\{0\}$ by Lemma 1.7. Thus the sum $R \oplus H$ is direct, and contains $H$ as a characteristic ideal, namely, the intersection of a commutator series. Since $R+H=R+R^{\perp}$ is an ideal of $L$, then $H$ is an ideal of $L$. Now we enlarge $H$ to a Levi complement $T=H+S$ for $R$ in $L$. Then $H$ centralizes $S$ and $R$, hence $K=R+S$. Since $B$ is perfect, Lemma 1.6 now shows that $K$ and $H$ are orthogonal that is that $H \cap H^{\perp}=\{0\}$. Our hypothesis now implies that $H=\{0\}$.

From Lemna 1.8 applied with $J=R^{\perp}$ we know $\left[R, R^{\perp}\right]=0$.

2.9. LEMMA. Under the circumstances of Lemma 2.8, let $S$ be a Levi complement for $R$ in $L$. Then $A=R^{\perp}+S$ is isomorphic to the inflation $I(S)$, and the restriction $q \mid(A \times A)$ is non-degenerate.

Proof. By Lemma 1.8, the $L$-module $R^{\perp}$ is isomorphic to the $L$-module $(L / R) *$. Since $L / R$ is semisimple and thus supports a non-degenerate invariant quadratic form, the $L$-modules $(L / R)^{*}$ and $L / R$ are isomorphic by Remark 1.1. Restriction of the operators fxom $L$ to $S$ shows that as $S$-modules, $L / R$ and $R^{\perp}$ are isomorphic. But the $S$-module $L / R$ is isomorphic to the $S$-module $|S|$ with the adjoint action. Thus $R^{\perp}$ is isomorphic to $|S|$ under the adjoint action. But this means precisely that $R^{\perp}+S$ is an inflation. 
By Lemma 2.7, then $R^{1}+S$ is a direct sum of inflations $I\left(S_{1}\right)+\ldots+I\left(S_{n}\right) \cdot$ By Lemma 1.6 , these summands are orthogonal to each other. Thus $q \mid(A \times A)$ is degenerate if and only if $q \mid\left(I\left(S_{j}\right) \times I\left(S_{j}\right)\right)$ is orthogonal for at least one $j$. If that were the case, then by Lemma 2.6 we would find a non-zero vector $x$ in the radical of $I\left(S_{j}\right)$, thus in particular in $R^{\perp}$, which would be orthogonal to all of $I\left(S_{j}\right)$ hence to all of $I\left(S_{1}\right)+\ldots+I\left(S_{n}\right)=R^{\perp}+S$. But since $x \in R^{\perp}$ it would also be orthogonal to $R$, hence to $R+S=L$ which is impossible since $q$ is non-degenerate.

Theorem $\mathrm{H}$ of the Introduction is now proved completely.

\section{References}

[1] N. Bourbaki, Groupes et algebres de Lie, Chap. 1, (Hermann, Paris, 1960) .

[2] J. Hilgert and K.H. Hofmann, "Lorentzian cones in Lie algebras", Monatshefte fur Mathematik (to appear) (Preprint Nr. 855 Oktober 1984, FB Mathematik, Technische Hochschule Darmstadt, 24 pp.)

[3] A.K. Guts and A.V. Levichev, "On the foundations of relativity theory", Soviet Math. Dokl. 30 (1984), 253-257.

[4] V.S. Keith, "On invariant bilinear forms on finite dimensional Lie algebras", Dissertation, Tulane University, New Orleans, 1984, 93 pp., University Microfilm International, P.O. Box 1764, Ann Arbor, Michigan 48106.

[5] J.L. Koszul, "Homologie et cohomologie des algèbres de Lie", Buz2. Soc. Math. France 78 (1950), 65-127.

[6] A. Medina, "Groupes de Lie munis de pseudométriques de Riemann biinvariantes", Séminaire de Gémetrie Diff., Exp. No. 6 M.R. 84C:53063 (Montpellier, 1981-82).

[7] A. Medina et $\mathrm{Ph}$. Revoy, "Algèbre de Lie et produit scalaire invariant", Séminaire de Géometrie Diff., (Montpellier, 1983-84). 
[8] A. Medina et Ph. Revoy, "Sur une géometrie Lorentzienne du groupe oszillateur", Seminaire de Geometrie Diff., (Montpellier, 1982-83).

[9] A. Medina et Ph. Revoy, "Caractérisation des groupes de Lie ayant une pseudométrique biinvariante, Applications, Travaux en cours", Séminaire Sud-Rhodanien de Géometrie III, (Hermann, Paris 1984).

[10] J. Milnor, "Curvatures of left invariant metrics on Lie groups", Advances in Math. 21 (1976), 283-329.

Fachbereich Mathematik,

Technische Hochschule Darmstadt,

Schlossgartenstr. 7,

D-6100 Darmstadt, Germany (FRG).

and

Department of Mathematics,

Tulane University,

New Orleans, La. 70118.

Added in proof. (November 27, 1985): A.A. Elashvili, Academy of Sciences of the Georgian SSR, Tbilisi, kindly informed us of the following sources relevant for the discussion in this article:

A.A. Astrakhantsev, Functional Analysis and its Appl. 19 (1985), 65-66.

A.A. Elashvili, "Frobenius Lie algebras II", Trans. Tbilisi Math. Inst. 77 (1985), 127-137. 